\title{
Self-reported tolerance influences prefrontal cortex hemodynamics and affective responses
}

\author{
Gavin Tempest ${ }^{1} \cdot$ Gaynor Parfitt $^{1}$
}

Published online: 3 September 2015

(C) Psychonomic Society, Inc. 2015

\begin{abstract}
The relationship between cognitive and sensory processes in the brain contributes to the regulation of affective responses (pleasure-displeasure). Exercise can be used to manipulate sensory processes (by increasing physiological demand) in order to examine the role of dispositional traits that may influence an individual's ability to cognitively regulate these responses. With the use of near infrared spectroscopy, in this study we examined the influence of self-reported tolerance upon prefrontal cortex (PFC) hemodynamics and affective responses. The hemodynamic response was measured in individuals with high or low tolerance during an incremental exercise test. Sensory manipulation was standardized against metabolic processes (ventilatory threshold [VT] and respiratory compensation point [RCP]), and affective responses were recorded. The results showed that the high-tolerance group displayed a larger hemodynamic response within the right PFC above VT (which increased above RCP). The lowtolerance group showed a larger hemodynamic response within the left PFC above VT. The high-tolerance group reported a more positive/less negative affective response above VT. These findings provide direct neurophysiological evidence of differential hemodynamic responses within the PFC that are associated with tolerance in the presence of increased physiological demands. This study supports the role of dispositional traits and previous theorizing into the underlying
\end{abstract}

Gavin Tempest

gavin.tempest@unisa.edu.au

1 Alliance for Research in Exercise, Nutrition and Activity (ARENA), Sansom Institute for Health Research, School of Health Sciences, University of South Australia, Adelaide, SA, Australia mechanisms (cognitive vs. sensory processes) of affective responses.

Keywords Near infrared spectroscopy · Prefrontal cortex · Dispositional trait $\cdot$ Exercise intensity

Affective responses (pleasure-displeasure) are proposed to be regulated in the brain by the prefrontal cortex (PFC) and subcortical regions, including the amygdala (Davidson \& Irwin, 1999). Research has shown an inverse coupling between PFC activation and activity in the amygdala (Hariri, Bookheimer, \& Mazziotta, 2000; Hariri, Mattay, Tessitore, Fera, \& Weinberger, 2003; Quirk, Likhtik, Pelletier, \& Paré, 2003). The relationship between the PFC and amygdala is strengthened when individuals are instructed to cognitively control negative (aversive) affective responses (Banks, Eddy, Angstadt, Nathan, \& Phan, 2007; Goldin, McRae, Ramel, \& Gross, 2008; Phan et al., 2005). A transient disruption, or reduced activation, of lateralized regions of the PFC is associated with a reduced ability to exert cognitive control to alleviate negative affective responses (Beauregard, 2007; Ochsner \& Gross, 2008; Ochsner et al., 2004).

During increased physiological demand (i.e., exercise), affective responses become less positive/more negative as the intensity of exercise increases (for reviews, see Ekkekakis, Parfitt, \& Petruzzello, 2011; Ekkekakis \& Petruzzello, 1999). According to a theoretical framework (the dual-mode model: Ekkekakis, 2003; Ekkekakis \& Acevedo, 2006), at intensities below the ventilatory threshold (VT; i.e., the point of transition from aerobic to anaerobic metabolism), individuals are able to maintain PFC activation to override the negative affective responses driven by sensory (interoceptive) input from the body. Interoceptive cues provide the brain with 
information from the body and include nociceptors (pain), metaboreceptors (chemical), thermoreceptors (temperature), mechanoreceptors, and baroreceptors (touch, pressure, tension; see Ament \& Verkerke, 2009). Above the VT (proximal to the respiratory compensation point $[\mathrm{RCP}]$; i.e., the point at which the physiological steady state cannot be maintained), competition between the PFC and subcortical regions, which receive sensory input from the body, becomes increasingly challenging. At this intensity, an individual's ability to maintain PFC activation becomes threatened. Recent work, using electroencephalography (EEG; Robertson \& Marino, 2015) and near infrared spectroscopy (NIRS; Jung, Moser, Baucsek, Dern, \& Schneider, 2015), has shown a redistribution of brain activation in PFC and motor regions as the intensity of exercise is increased. Since exercise influences PFC activation, it may be used to manipulate sensory input from the body (presumably to the amygdala) in order to examine the role of the PFC in the regulation of affective responses.

One potential factor that may impact PFC activation, and therefore an individual's level of cognitive control to regulate affective responses, is the role of dispositional traits. The Preference for and Tolerance of Exercise Intensity Questionnaire (PRETIE-Q) is an inventory created to assess arousability and sensory-modulation-related traits for interoceptive (as opposed to exteroceptive) stimuli (see Ekkekakis, Hall, \& Petruzzello, 2005, for full details). The authors define preference as "a predisposition to select a particular level of exercise intensity when given the opportunity" (p. 354), and tolerance as "a trait that influences one's ability to continue exercising at an imposed level of intensity even when the activity becomes uncomfortable or unpleasant" (p. 354). The identification of preference and tolerance as dispositional traits is supported by no change in these scores after a 6week training program that resulted in improvements in physical fitness (Hall, Petruzzello, Ekkekakis, Miller, \& Bixby, 2014). Conceptual validation of the scales (Ekkekakis et al., 2005) showed that neither preference nor tolerance was associated with affective responses reported during exercise at intensities below VT. However, both scales were positively associated with affective responses at VT, but only the tolerance scale related to affective responses above VT (Ekkekakis et al., 2005; Ekkekakis, Lind, Hall, \& Petruzzello, 2007). Therefore, a higher tolerance was associated with a more positive affective response at intensities of exercise above the VT, which is predominantly aversive or negative (Ekkekakis et al., 2011).

It is proposed that tolerance influences affective responses through cognitive processes in the PFC (Ekkekakis et al., 2005). Consistent with the dual-mode model, sensory input to the body is biologically hard-wired; however, the perception and modulation of the interoceptive input by the PFC may be influenced by predisposed traits. In other words, individuals with high tolerance may be more capable of cognitively regulating how sensory information from the body is perceived during exercise at physiologically challenging intensities (i.e., above VT) than those with low tolerance. Individuals with high tolerance would report less-negative affective responses than those with low tolerance. In addition, individuals with high tolerance may exercise for longer at an imposed level of intensity (i.e., above VT) that is uncomfortable or unpleasant (Ekkekakis et al., 2005; Ekkekakis et al., 2007).

The present study investigates whether tolerance of the intensity of exercise influences PFC hemodynamics and affective responses during incremental exercise. Bilateral measures of the PFC (right and left) were measured because of evidence that lateralization is involved in cognitive-control processes (Ochsner et al., 2004). Prefrontal hemodynamics can be recorded by a noninvasive neuroimaging technique, NIRS, that is suitable for use during exercise (Ferrari \& Quaresima, 2012; Perrey, 2012). Changes $(\Delta)$ in cerebral hemodynamics - oxygenation $\left(\mathrm{O}_{2} \mathrm{Hb}\right.$; oxygen delivery and blood flow), deoxygenation ( $\mathrm{HHb}$; oxygen extraction), and total blood volume $\left(\mathrm{tHb}=\mathrm{O}_{2} \mathrm{Hb}+\mathrm{HHb}\right)$-reflect metabolic changes associated with functional (neural) activation and metabolism (Perrey, 2012). Cerebral activation is defined by a slight decrease in oxygen extraction ( $\mathrm{HHb})$ and an increase in oxygenation $\left(\mathrm{O}_{2} \mathrm{Hb}\right.$; two-fold of $\left.\mathrm{HHb}\right)$, leading to hyperoxygenation (i.e., greater oxygen availability; Perrey, 2012). A meta-analysis of studies examining the hemodynamic response using NIRS during exercise showed a quadratic trend of cerebral oxygenation as the exercise increased: Oxygenation increased from low to moderate and remained stable from moderate to hard, but declined at very hard (approximately RCP to exhaustion) intensities (Rooks, Thom, McCully, \& Dishman, 2010). However, the authors indicated that the decline near exhaustion was less pronounced in trained, as opposed to untrained, individuals.

Three hypotheses were examined here: (1) Prefrontal cortex (PFC) hemodynamics are different between individuals with high or low tolerance; (2) individuals with high tolerance report less-negative affective responses at intensities above VT; and (3) individuals with high tolerance exercise for longer than those with low tolerance at intensities above VT.

\section{Method}

\section{Participants}

A university cohort of sports science students $(n=259)$ completed the PRETIE-Q (Ekkekakis et al., 2005) during a timetabled session. Following quantitative analysis of hemodynamic variables $\left(\Delta \mathrm{O}_{2} \mathrm{Hb}\right.$ and $\left.\Delta \mathrm{HHb}\right)$ measured during exercise intensities up to exhaustion with an optode (transmitterdetector) distance of $4 \mathrm{~cm}$ (Rooks et al., 2010; assuming statistical power $=.80$ and $\alpha=.05$ ), and allowing for attrition, at 
least 14 participants were required per group. Therefore, participants with the highest and lowest tolerance scores were invited to participate in the study ( $n=28$; see Table 1 in the Results). All except two participants were right-handed. The volunteers read and signed an informed consent form that was approved by the departmental Ethics Committee. The Physical Activity Readiness Questionnaire (Canadian Society for Exercise Physiology, 2002) was used to ensure suitability to exercise.

\section{Measures}

Preference for and Tolerance of the Intensity of Exercise Questionnaire Tolerance was assessed using the PRETIE-Q (Ekkekakis et al., 2005). The 16-item questionnaire contains eight items for preference (four high, four low) and, similarly, eight items for tolerance (four high, four low). Each item comprises a five-point response scale $(1=I$ totally disagree, $2=I$ disagree, $3=$ Neither agree nor disagree, $4=$ I agree, and 5 = I strongly agree). The alpha coefficients of internal consistency ranged from .82 to .87 for the Tolerance scale, and it displayed test-retest reliability coefficients of .85 and .72 after 3- and 4-month intervals (see Ekkekakis et al., 2005, for the complete questionnaire). The alpha coefficients of internal consistency in the present study ranged between .76 and .81 for the tolerance scores of the high- and low-tolerance groups.

Near infrared spectroscopy Cerebral hemodynamics were measured using NIRS (NIRO 200 Hamamatsu Photonics, Hamamatsu, Japan). The emitter and detector were encased in a rubber holder with a separation distance of $4 \mathrm{~cm}$. A differential path length factor of 5.93 for the adult forehead was used (van der Zee et al., 1992) to provide a measure of concentration changes $(\Delta)$ in micromolar $(\mu \mathrm{M})$ units of $\mathrm{O}_{2} \mathrm{Hb}$, $\mathrm{HHb}$, and $\mathrm{tHb}$ (sample rate: $2 \mathrm{~Hz}$ ). The probes were placed approximately over the left and right dorsolateral PFC (between Fp1-F3 and Fp2-F4, respectively, of the International 10-20 System for EEG electrode placement) and were secured to the skin using a double adhesive sticker. Elastic surgical tape (Kinesio Tex Gold) and a dark bandage were placed over the holders around the forehead.

The Feeling Scale Affective responses were measured using the Feeling Scale (Hardy \& Rejeski, 1989). The unidimensional 11-point scale (ranging from -5 to +5 , with verbal anchors at all odd integers and at the zero point: -5 very bad, -3 bad, -1 fairly bad, 0 neutral, 1 fairly good, 3 good, 5 very good) allows multiple assessments to be made during exercise. The Feeling Scale corresponds to one of the two dimensions of the circumplex model of feeling states (Russell, Weiss, \& Mendelsohn, 1989) and is recommended for measuring basic affect (pleasure-displeasure; Ekkekakis \& Petruzzello, 1999).

\section{Procedures}

The study required individuals to visit the exercise physiology laboratory (approximate temperature of $24{ }^{\circ} \mathrm{C}$ and relative humidity of $40 \%$ ) on one occasion. Upon arrival, participants completed the informed consent form and exercise testing questionnaire, and initial assessments were taken (age, height, and body mass). The procedures for the exercise tests were explained and a description of the Feeling Scale was provided. Participants were seated on a recumbent cycle ergometer (Lode Angio, Groningen, the Netherlands), and the NIRS optodes were carefully positioned. A facemask was fitted in order to measure metabolic data via online gas analysis (Cortex Metalyzer 3B, Biophysik, Leipzig, Germany). The participants then completed an incremental $\left(20 \mathrm{~W} \mathrm{~min}^{-1}\right.$, pedal cadence $70 \mathrm{rpm}$ ) cycling exercise test to exhaustion. The end of the test was determined by volitional cessation of exercise or failure to maintain pedal cadence despite strong verbal encouragement. The achievement of $\dot{\mathrm{VO}}_{2 \text { peak }}$ was verified by (a) a peak or plateau in oxygen consumption (changes $<$ $2 \mathrm{ml} \cdot \mathrm{kg}^{-1} \cdot \mathrm{min}^{-1}$ ) with increasing workload and (b) a respiratory exchange ratio of at least 1.10 . Cerebral hemodynamic responses and expired gases were measured continuously, and affective responses were recorded before, during (every minute), and at the end of exercise.

\section{Data and statistical analyses}

The peak oxygen uptake $\left(\dot{\mathrm{V}} \mathrm{O}_{2 \text { peak }}\right)$ was determined by the highest 30 -s average of oxygen uptake $\left(\dot{\mathrm{V}} \mathrm{O}_{2}\right.$, measured in $\left.\mathrm{ml} \mathrm{kg}^{-1} \min ^{-1}\right)$. The VT was determined using the threemethod procedure proposed by Gaskill et al. (2001), and the RCP was determined according to Beaver, Wasserman, and Whipp (1986).

Thirty-second baseline measures of cerebral hemodynamics $\left(\Delta \mathrm{O}_{2} \mathrm{Hb}, \Delta \mathrm{HHb}\right.$, and $\left.\Delta \mathrm{tHb}\right)$ were recorded prior to exercise. The data were exported every $10 \mathrm{~s}$ and normalized to express the magnitude of changes from the baseline period (arbitrarily defined as $0 \mu \mathrm{M}$ ) at the start of exercise. Cerebral hemodynamic variables were selected at time points corresponding to intensities of exercise: $80 \%$ of VT (below VT), $V T, R C P$, and end of exercise (end). Affective responses were extracted at time points corresponding to pre-exercise and to each of the intensities of exercise.

To examine Hypothesis 1, a Group (2: high, low) $\times$ Hemisphere (2: right, left PFC) $\times$ Time (4: below VT, VT, $\mathrm{RCP}$, end) mixed-model analysis of variance (ANOVA) was conducted for each of the NIRS variables $\left(\Delta \mathrm{O}_{2} \mathrm{Hb}, \Delta \mathrm{HHb}\right.$, and $\Delta \mathrm{tHb})$. To examine Hypothesis 2, a Group (2: high, low) $\times$ Time (5: pre-exercise, below VT, VT, RCP, end) mixed-model 
Table 1 Participant demographics and average 30-s oxygen uptake $\left(\mathrm{V}_{2}\right)$ for the high- and low-tolerance groups, shown as means (and standard deviations)

\begin{tabular}{|c|c|c|c|c|c|c|}
\hline & \multicolumn{3}{|c|}{ High-Tolerance Group } & \multicolumn{3}{|c|}{ Low-Tolerance Group } \\
\hline & $\operatorname{Males}^{\#}(n=7)$ & Females $(n=7)$ & Total $^{*}(n=14)$ & $\operatorname{Males}^{\#}(n=7)$ & Females $(n=7)$ & Total $^{*}(n=14)$ \\
\hline \multicolumn{7}{|l|}{ Demographics } \\
\hline Age (yrs) & $20.1(1.1)$ & $20.7(1.4)$ & $20.6(1.4)$ & $21.3(2.7)$ & $20.8(3.8)$ & $21.5(3.4)$ \\
\hline Height $(\mathrm{cm})$ & $177.1(5.0)$ & $164.8(3.1)$ & $171.0(7.5)$ & $179.0(8.4)$ & $167.4(5.5)$ & $173.2(9.1)$ \\
\hline Body mass (kg) & $71.5(4.1)$ & $65.0(7.1)$ & $69.3(7.3)$ & $77.6(9.1)$ & $57.5(3.8)$ & $68.6(12.8)$ \\
\hline BMI & $22.8(1.4)$ & $23.9(2.5)$ & $23.4(2.0)$ & $24.2(2.2)$ & $20.6(1.9)$ & $22.4(2.7)$ \\
\hline \multicolumn{7}{|c|}{$\dot{\mathrm{V}} \mathrm{O}_{2}\left(\mathrm{ml} \cdot \mathrm{kg}^{-1} \cdot \mathrm{min}^{-1}\right)$} \\
\hline Below VT & $20.9(2.8)$ & $18.1(1.7)$ & $19.5(2.7)$ & $18.8(3.2)$ & $18.0(1.6)$ & $18.4(2.5)$ \\
\hline VT & $26.1(3.5)$ & $22.7(2.3)$ & $24.4(3.4)$ & $23.6(4.0)$ & $22.5(2.0)$ & $23.0(3.1)$ \\
\hline $\mathrm{RCP}$ & $40.1(4.7)$ & $34.8(3.5)$ & $37.8(5.1)$ & $36.7(7.0)$ & $34.2(3.6)$ & $35.4(5.5)$ \\
\hline End $\left(\dot{\mathrm{V}} \mathrm{O}_{2 \text { peak }}\right)$ & $49.4(5.9)$ & $41.7(4.2)$ & $45.6(6.3)$ & $43.0(8.2)$ & $41.1(4.0)$ & $42.1(6.3)$ \\
\hline
\end{tabular}

Oxygen uptake was measured at time points corresponding to $80 \%$ of ventilatory threshold (below VT), at VT, the respiratory compensation point (RCP), and end of exercise (End), indicating maximal oxygen uptake $\left(\dot{\mathrm{V}} \mathrm{O}_{2 \text { peak }}\right) .{ }^{*}$ Significant increase in $\dot{\mathrm{VO}}_{2}$ at each time point. ${ }^{*}$ Significantly higher $\dot{\mathrm{V}} \mathrm{O}_{2}$ in males than in females at each time point $(p<.05)$

ANOVA was conducted on the affective responses. Finally, to examine Hypothesis 3, two independent-sample $t$ tests, with Bonferroni correction (alpha of .05/2 [number of tests] = .025), were conducted for the durations of exercise (from VT to RCP and from RCP to end) of the high- and lowtolerance groups. All statistical analyses were performed using SPSS version 18.0 (IBM Corp., Armonk, NY, USA). Greenhouse-Geisser corrections were applied if the assumption of sphericity was not met. All significant main and interaction effects $(p<.05)$ were followed by Bonferroniadjusted pairwise comparisons and simple main effects tests. Effect sizes associated with the $F$ statistics (ANOVAs) were expressed as partial eta-squared $\left(\eta_{\mathrm{p}}{ }^{2}\right)$, defined as small (.01), medium (.06), and large (.14) (Cohen, Dunbar, \& McClelland, 1990). Values are expressed as means $\pm S D$ s unless otherwise stated.

\section{Results}

\section{Preliminary analyses}

To ensure that tolerance scores were different between the two groups (high, low) and to verify whether there was a difference in overall times to exhaustion, two independent $t$ tests were conducted. These tests showed that tolerance scores differed significantly between the high- $(M=33.1, S D=2.3$, range 30 to 38$)$ and low- $(M=21.1, S D=1.9$, range 18 to $24)$ tolerance groups, $t(26)=15.07, p<.001$. No difference in overall times to exhaustion (in seconds) was apparent between the high- $(M=676, S D=116)$ and low- $(M=646, S D=93)$ tolerance groups $(p>.05)$.

To examine any differences in fitness (indicated by $\dot{\mathrm{VO}}_{2}$, the average 30-s oxygen uptake) between the males and females in the high- and low-tolerance groups at time points corresponding to different intensities of exercise (below VT, VT, RCP, and end), a Group (2) × Gender (2) × Time (4) mixed-model ANOVA was conducted. The analysis showed no differences in $\dot{\mathrm{V}}_{2}$ between groups (main and interaction effects; $p>.05$ ). However, as expected, $\dot{\mathrm{O}}_{2}$ was (a) higher in males $(M=32.4, S D=5.1)$ than in females $(M=29.1, S D=$ $2.7), F(1,24)=4.73, p<.05, \eta_{\mathrm{p}}{ }^{2}=.17$, and (b) significantly different at each intensity: below VT $(M=19.0, S D=2.7)$, VT $(M=23.7, S D=3.2), \operatorname{RCP}(M=36.1, S D=5.3)$, and end of exercise $(M=43.8, S D=6.4), F(1,26)=1,141.48, p<.001$, $\eta_{\mathrm{p}}{ }^{2}=.98$. A Gender $\times$ Time interaction indicated that males had a disproportionately higher $\dot{\mathrm{O}}_{2}$ at RCP than did females, $F(1,26)=4.52, p<.05, \eta_{\mathrm{p}}{ }^{2}=.16$ (see Table 1$)$.

\section{Cerebral hemodynamics (Hypothesis 1)}

As a result of the Gender $\times$ Time interaction for $\dot{\mathrm{V}}_{2}$, preliminary analyses of covariance were conducted for $\Delta \mathrm{O}_{2} \mathrm{Hb}$, $\Delta \mathrm{HHb}$, and $\Delta \mathrm{tHb}$, in order to include gender as a covariate. Additional analyses were also conducted using handedness as a covariate. The analyses showed no influence of gender or handedness upon the significant main and interaction effects for group, hemisphere, and/or time ( $p$ s $>.05)$. However, as expected, significant Gender $\times$ Time interactions were recorded for $\Delta \mathrm{O}_{2} \mathrm{Hb}$ and $\Delta \mathrm{tHb}$ (males were higher than females at 
$\mathrm{RCP}, p<.05)$. The ANOVA results and the unadjusted means for $\Delta \mathrm{O}_{2} \mathrm{Hb}, \Delta \mathrm{HHb}$, and $\Delta \mathrm{tHb}$ are presented.

\section{Cerebral oxygenation $\left(\Delta \mathrm{O}_{2} \mathrm{Hb}\right)$}

A significant main effect of time, $F(1,33)=99.39, p<.001$, $\eta_{\mathrm{p}}{ }^{2}=.79$, and significant Group $\times$ Hemisphere, $F(1,26)=$ $4.37, p=.047, \eta_{\mathrm{p}}{ }^{2}=.14$, and Group $\times$ Hemisphere $\times$ Time, $F(1,34)=4.14, p=.039, \eta_{\mathrm{p}}{ }^{2}=.14$, interactions were recorded for $\Delta \mathrm{O}_{2} \mathrm{Hb}(\mu \mathrm{M})$. Cerebral $\Delta \mathrm{O}_{2} \mathrm{Hb}$ remained stable from below VT $(M=2.04, S D=2.39)$ to VT $(M=2.71, S D=2.82)$, then increased from VT to RCP $(M=12.14, S D=5.51)$ and from RCP to end $(M=13.49, S D=6.87)$ in both groups. In the left PFC, $\Delta \mathrm{O}_{2} \mathrm{Hb}$ was larger in the low-tolerance $(M=8.33$, $S D=3.88)$ than in the high-tolerance $(M=6.80, S D=4.36)$ group. In the right PFC, $\Delta \mathrm{O}_{2} \mathrm{Hb}$ was similar between the lowtolerance $(M=7.46, S D=4.01)$ and high-tolerance $(M=7.78$, $S D=4.27$ ) groups. The three-factor interaction indicated that from VT to RCP, the low-tolerance group had a significantly larger $\Delta \mathrm{O}_{2} \mathrm{Hb}$ in the left than in the right PFC at RCP (and when compared to both hemispheres in the high-tolerance group). From RCP to end, $\Delta \mathrm{O}_{2} \mathrm{Hb}$ remained stable in both hemispheres in the low-tolerance group, but significantly increased in both hemispheres in the high-tolerance group. Therefore, at the end, $\Delta \mathrm{O}_{2} \mathrm{Hb}$ remained larger in the left than in the right $\mathrm{PFC}$ in the low-tolerance group, whereas $\Delta \mathrm{O}_{2} \mathrm{Hb}$ was larger in the right than in the left PFC in the hightolerance group (see Fig. 1).

\section{Cerebral deoxygenation $(\Delta \mathrm{HHb})$}

Significant main effects of hemisphere, $F(1,26)=5.33, p=$ $.029, \eta_{\mathrm{p}}{ }^{2}=.17$, and time, $F(1,31)=50.99, p<.001, \eta_{\mathrm{p}}{ }^{2}=.66$, were recorded for $\Delta \mathrm{HHb}(\mu \mathrm{M})$. Follow-up tests indicated that $\Delta \mathrm{HHb}$ was larger in the right $(M=1.32, S D=1.23)$ than in the left $(M=1.01, S D=1.32)$ PFC in both groups. Cerebral $\triangle \mathrm{HHb}$ remained stable from below VT $(M=0.16, S D=$ $0.53)$ to VT $(M=0.06, S D=0.68)$, then increased from VT to $\operatorname{RCP}(M=1.03, S D=1.68)$ and from RCP to end $(M=3.41$, $S D=2.53)$ in both groups.

\section{Cerebral blood volume $(\Delta \mathrm{tHb})$}

A significant main effect of time, $F(1,32)=128.66, p<.001$, $\eta_{\mathrm{p}}{ }^{2}=.83$, and a Group $\times$ Hemisphere $\times$ Time interaction, $F(1$, 37) $=3.67, p=.049, \eta_{\mathrm{p}}{ }^{2}=.12$, were recorded for $\Delta \mathrm{tHb}(\mu \mathrm{M})$. Cerebral $\Delta \mathrm{tHb}$ remained stable from below VT $(M=2.20, S D$ $=2.33)$ to VT $(M=2.77, S D=2.80)$, then increased from VT to RCP $(M=13.16, S D=6.26)$ and from RCP to end $(M=$ $16.89, S D=7.50$ ) in both groups. The three-factor interaction indicated that from VT to RCP, $\Delta \mathrm{tHb}$ was significantly smaller in the left PFC in the high-tolerance than in the low-tolerance group. From RCP to end, $\Delta \mathrm{tHb}$ was larger in the high- tolerance than in the low-tolerance group. At the end, $\Delta \mathrm{tHb}$ remained similar in both hemispheres in the low-tolerance group, whereas it was larger in the right than in the left PFC in the high-tolerance group (and when compared to both hemispheres in the low-tolerance group; see Fig. 1).

\section{Affective responses (Hypothesis 2)}

A significant main effect of time, $F(2,39)=38.35, p<.001$, $\eta_{\mathrm{p}}{ }^{2}=.56$, and a significant Group $\times$ Time interaction, $F(4$, $104)=3.57, p=.009, \eta_{\mathrm{p}}{ }^{2}=.05$, were recorded for affective responses. Affective responses declined from pre-exercise $(M$ $=2.43, S D=1.35)$ to below VT $(M=1.86, S D=1.24)$, remained stable until VT $(M=1.68, S D=1.44)$, and declined from VT to RCP $(M=-0.11, S D=2.04)$ and from RCP to end $(M=-1.04, S D=2.52)$. The interaction indicated that the decline in affective responses from pre-exercise to below VT was a result of the responses in the low-tolerance group. Both groups reported similar affective responses from below VT to VT, at which point we observed a larger decline in the low- than in the high-tolerance group. Therefore, despite positive affective responses in both groups at VT, affective responses were negative at RCP and end in the low-tolerance group (see Fig. 2).

\section{Exercise duration (Hypothesis 3)}

There was no difference in the durations of exercise (in seconds) from VT to RCP between the high- $(M=250, S D=44)$ and low- $(M=260, S D=53)$ tolerance groups $(p>.025)$. However, the duration of exercise from RCP to end was significantly longer in the high- $(M=145, S D=39)$ than in the low- $(M=113, S D=24)$ tolerance group, $t(26)=2.62, p<$ .025. A significant positive correlation was shown between the duration of exercise from RCP to end and the tolerance scores $(r=.48, p<.001)$.

\section{Discussion}

Exercise was used to manipulate sensory processes (by increasing physiological demand) in order to examine the influence of a dispositional trait upon PFC hemodynamics and affective responses. Tolerance has been proposed to influence an individual's ability to cognitively regulate affective responses. In addition, tolerance impacts an individual's ability to continue exercising at levels of intensity associated with feelings of displeasure and discomfort (Ekkekakis et al., 2005; Ekkekakis et al., 2007). This study has provided three main findings. First, individuals with high and low tolerance showed asymmetrical PFC hemodynamics during exercise at intensities above VT (Hypothesis 1). Second, low-tolerance individuals reported negative affective responses at intensities 
Fig. 1 Change in cerebral (a) oxygenation $\left(\Delta \mathrm{O}_{2} \mathrm{Hb}\right),(\mathbf{b})$ deoxygenation $(\triangle \mathrm{HHb})$, and (c) blood volume $(\Delta \mathrm{tHb})$ in the right and left prefrontal cortices at each time point for the high- and lowtolerance groups, shown as means and SEMs. The time points correspond to $80 \%$ of ventilatory threshold (below VT), VT, the respiratory compensation point (RCP), and the end of exercise (End). * Significantly higher than the other hemisphere within a group. ${ }^{* *}$ Significantly higher than the other hemisphere within and between groups. "Significantly higher than the same hemisphere between groups $(p<.05)$. The patterns of the hemodynamic data $\left(\Delta \mathrm{O}_{2} \mathrm{Hb}, \Delta \mathrm{HHb}\right.$, and $\left.\Delta \mathrm{tHb}\right)$ were relatively linear between the time points examined

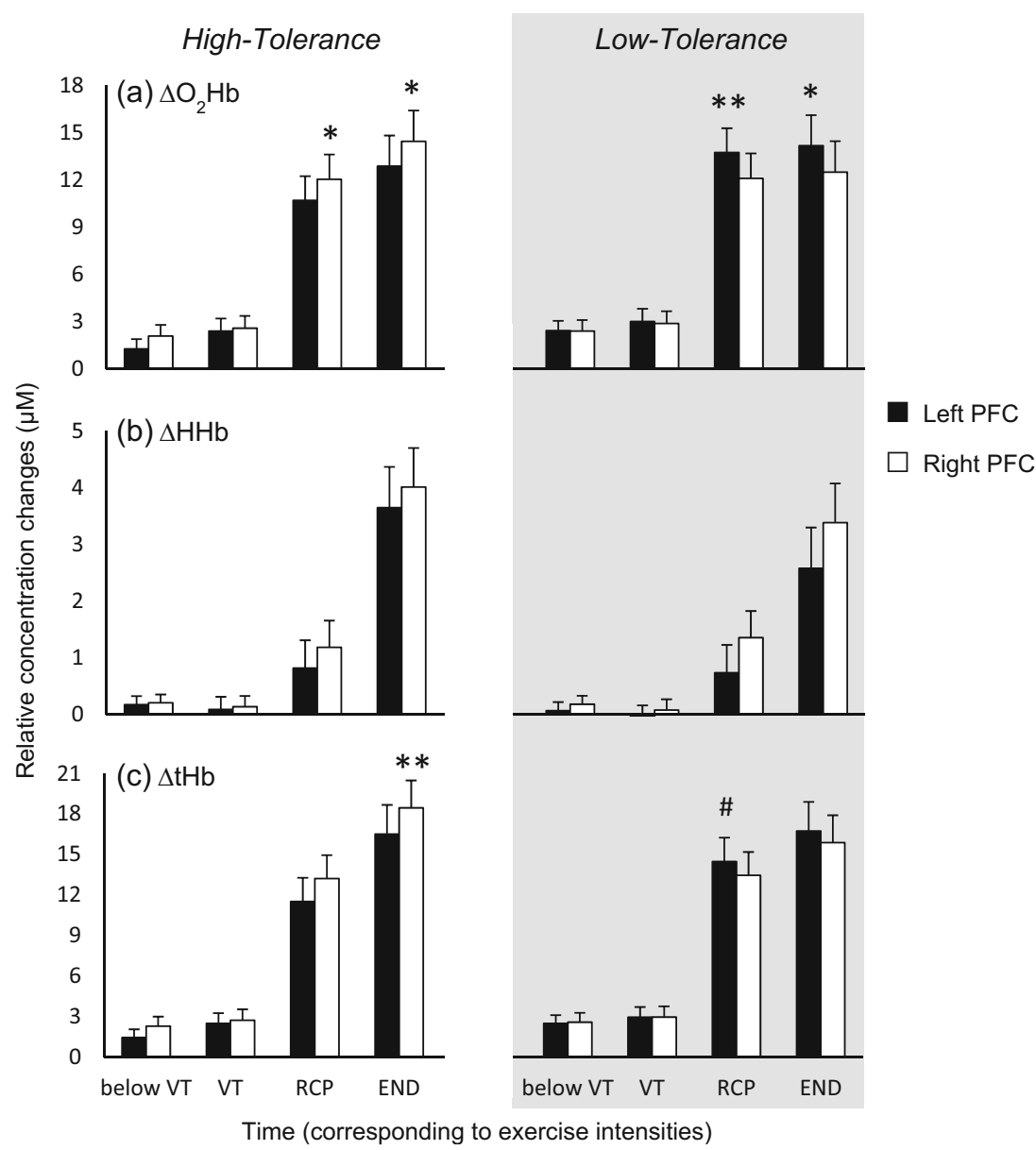

of exercise above VT (Hypothesis 2). Finally, despite a lack of differences in fitness (i.e., in $\dot{\mathrm{V}}_{2}$ ) between the two groups, individuals with high tolerance exercised for longer above their individually determined RCP than did those with low tolerance (Hypothesis 3).

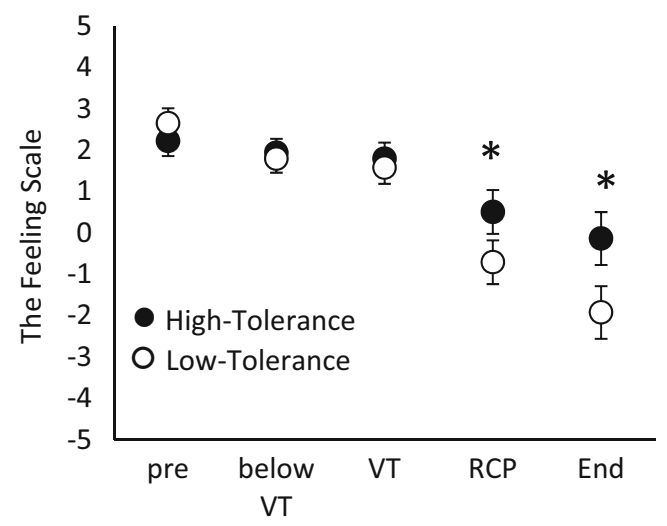

Fig. 2 Affective responses at each time point for the high- and lowtolerance groups, shown as means and SEMs. The time points correspond to pre-exercise (pre), $80 \%$ of the ventilatory threshold (below VT), VT, the respiratory compensation point (RCP), and the end of exercise (End). *Significantly more positive affective responses in the high- than in the low-tolerance group $(p<.05)$
In line with the dual-mode model, it has been proposed that active involvement of the PFC suppresses aversive stimuli theorized to be mediated by the amygdala, driven by intensified sensory (interoceptive) input from the body (Ekkekakis, 2003; Ekkekakis \& Acevedo, 2006). At intensities below VT, no differences in PFC hemodynamics have been observed between the high- and low-tolerance groups, and affective responses were positive. According to the model, sensory input from the body does not threaten homeostasis, and affective responses are predominantly positive. Tolerance was not expected to influence PFC hemodynamics and has not previously been shown to correlate with affective responses below VT (Ekkekakis et al., 2005).

At intensities from VT to RCP, we found no differences in cerebral blood flow and volume in the right PFC between the high- and low-tolerance groups. However, in the left PFC, the low-tolerance group showed greater blood flow $\left(\Delta \mathrm{O}_{2} \mathrm{Hb}\right)$ and volume $(\Delta \mathrm{tHb})$ than the high-tolerance group. This implies that the hemodynamic response in the left PFC in the lowtolerance group was larger than those observed in the hightolerance group at RCP due to the increase in oxygen availability. Oxygen extraction $(\Delta \mathrm{HHb})$ increased in both hemispheres in both groups. Affective responses were negative in 
the low-tolerance group, but remained positive in the hightolerance group. Therefore, in the low-tolerance group a larger hemodynamic response in the left PFC may have been required to maintain cognitive-control processes as the intensity of exercise started to become challenging (Ekkekakis, 2003; Ekkekakis \& Acevedo, 2006). This would allow the individual to continue exercise despite the presence of negative affective responses (Ekkekakis et al., 2005).

At intensities from RCP to end, we observed differences in cerebral blood flow and volume in both hemispheres between the high- and low-tolerance groups. Individuals with low tolerance had a smaller hemodynamic response at intensities above the RCP. This was shown by stable cerebral blood flow $\left(\Delta \mathrm{O}_{2} \mathrm{Hb}\right)$ in the presence of increases in oxygen extraction ( $\triangle \mathrm{HHb}$ - i.e., a lack of oxygen supply relative to demand). Individuals with high tolerance had an increased hemodynamic response at intensities above RCP. This was shown by increases in cerebral blood flow $\left(\Delta \mathrm{O}_{2} \mathrm{Hb}\right)$ and oxygen extraction $(\Delta \mathrm{HHb}$ - i.e., an adequate oxygen supply relative to demand). The low-tolerance group showed deregulation (hypofrontality effects; Dietrich, 2003, 2006) in the PFC and reported a more negative affective response than did the high-tolerance group. In addition, the duration of exercise was positively associated with tolerance scores at these intensities (RCP to end). The high-tolerance group showed an ability to continue exercise at a level of intensity associated with feelings of displeasure and discomfort (Ekkekakis et al., 2005; Ekkekakis et al., 2007). However, due to the larger hemodynamic response in the high-tolerance group, it may be that these individuals were able to maintain PFC function more efficiently because their affective responses are not as negative.

Consistent with the dual-mode model, the intensified sensory (interoceptive) input, presumably to the amygdala, provided a greater challenge to those individuals with low tolerance to maintain PFC activation (Ekkekakis, 2003; Ekkekakis $\&$ Acevedo, 2006). As was previously reported, affective responses were less positive/negative at intensities above VT (Ekkekakis et al., 2011), but these were distinctly more negative in the low- than in the high-tolerance group. Therefore, tolerance potentially influenced the individual's ability to exert cognitive control in the attempt to alleviate negative affective responses (Beauregard, 2007; Ochsner et al., 2004; Ochsner \& Gross, 2008).

Asymmetrical PFC hemodynamics were shown between the high- and low-tolerance groups. Previous research investigating affective and motivational (approach-avoidance) processes using EEG had indicated that avoidance/withdrawalrelated behaviors are associated with greater activity within the right relative to the left PFC (Davidson, 1993). However, the larger hemodynamic response within the right PFC observed in those individuals with high tolerance presumably does not reflect withdrawal-type behavior, in light of their perseverance with exercise at intensities above the RCP. We postulated that, in this study, the larger hemodynamic response (reflecting greater activity) in the right PFC was potentially due to active avoidance of withdrawal-type behavior (as opposed to promoting approach-related behavior-i.e., greater activity in the left PFC) in the presence of negative affect (Woo, Kim, Kim, Petruzzello, \& Hatfield, 2009). This would allow individuals with high tolerance to continue exercising and indicates differential cognitive and motivational processes from those used by individuals with low tolerance.

Alternatively, Craig's (2005) theory of forebrain emotional asymmetry may explain the greater hemodynamic response within the right $\mathrm{PFC}$ of the individuals with high tolerance. Craig proposed the lateralization of interoceptive input from afferent cues. Parasympathetic afferents activate primarily the left, and sympathetic afferents the right, insular cortex. Due to the increased sympathetic activity that exercise induces, heightened activation of the right insula may have had an impact on the overlying regions of the right PFC measured in this study (Woo et al., 2009). Although we observed no physiological differences between the two groups, the perceptual representation of the afferent input may have been influenced by the individual's tolerance of the intensity of exercise (Ekkekakis et al., 2005). It is plausible that the individuals with high tolerance had a more acute ability to regulate their somatosensory perception.

It is important to highlight a range of factors that may have contributed to the differences in hemodynamic and perceptual responses between the high- and low-tolerance groups. In this cross-sectional study, the training status of the participants was not assessed; however, as was previously indicated, no differences in aerobic power between the two groups were shown. Rooks et al. (2010) showed a decline in cerebral oxygenation at very hard intensities (i.e., above RCP) that was influenced by training status. In the present study, no decline in cerebral oxygenation was observed at a similar intensity; instead, cerebral oxygenation increased in the high-tolerance and remained stable in the low-tolerance group. The lack of a drop in PFC oxygenation near exhaustion has recently been observed by others (Jung et al., 2015). A potential explanation of this discrepancy is that it may be due to differences in the placement of the optodes over the PFC (Jung et al., 2015), since prior work has shown region-specific changes in PFC hemodynamics (Tempest, Eston, \& Parfitt, 2014). Finally, both males and females took part in this study, and no gender effects were observed (other than those likely explained by fitness). Future research should interrogate the potential influences or relationship of training status, gender, and tolerance upon hemodynamic responses.

The influence of the dispositional trait of tolerance upon PFC hemodynamics has been highlighted in this study. However, no measures of the exact cognitive-control mechanisms proposed have been assessed, nor have measures of 
subcortical areas of the brain (i.e., the amygdala) been recorded. Future studies should utilize study designs from emotion research (i.e., to include appraisal/suppression paradigms) so as to understand an individual's cognitive and motivational strategies involved in the regulation of affective responses during increased physiological demand (such as exercise). Prior work has shown that the use of imagery as a cognitive strategy can maintain activation (larger hemodynamic response) of the PFC and that it results in a more positive affective response at intensities above VT, but not at RCP (Tempest $\&$ Parfitt, 2013). In addition, the relationship between oxygenation in different regions (right-left, dorsal-ventral) of the $\mathrm{PFC}$ and affective responses is influenced by increases in the intensity of exercise above VT (Tempest et al., 2014).

\section{Summary}

This study used methods to standardize sensory input from the body induced by exercise in order to investigate the regulation of affective responses in the PFC. Tolerance is a dispositional trait associated with cerebral blood flow during exercise. It appears that those individuals with low tolerance may utilize cognitive mechanisms in the left PFC during exercise at intensities above the VT and proximal to the RCP. Those individuals with high tolerance do not appear to utilize the left PFC until higher intensities of exercise (i.e., above RCP). Additionally, individuals with high tolerance induce a greater hemodynamic response in the right PFC during exercise at intensities near exhaustion. Tolerance potentially influences, or is influenced by, the way that an individual perceives and self-regulates somatosensory information during exercise. We propose that tolerance influences the interplay between cognitive and sensory processes that influences the regulation of affective responses.

Author note The authors would like to thank Claire Broomfield and Laura Drewell for their assistance in participant recruitment and data collection and Panteleimon Ekkekakis for his advice on earlier drafts of the manuscript.

\section{References}

Ament, W., \& Verkerke, G. J. (2009). Exercise and fatigue. Sports Medicine, 39, 389-422.

Banks, S. J., Eddy, K. T., Angstadt, M., Nathan, P. J., \& Phan, K. L. (2007). Amygdala-frontal connectivity during emotion regulation. Social Cognitive and Affective Neuroscience, 2, 303-312.

Beauregard, M. (2007). Mind does really matter: Evidence from neuroimaging studies of emotional self-regulation, psychotherapy, and placebo effect. Progress in Neurobiology, 81, 218-236.

Beaver, W. L., Wasserman, K., \& Whipp, B. J. (1986). A new method for detecting anaerobic threshold by gas exchange. Journal of Applied Physiology, 60, 2020-2027.
Canadian Society for Exercise Physiology. (2002). Physical Activity Readiness Questionnaire (2002 revision). Retrieved from www. csep.ca/cmfiles/publications/parq/par-q.pdf

Cohen, J. D., Dunbar, K., \& McClelland, J. L. (1990). On the control of automatic processes: A parallel distributed processing account of the Stroop effect. Psychological Review, 97, 332-361. doi:10.1037/ 0033-295X.97.3.332

Craig, A. D. (2005). Forebrain emotional asymmetry: A neuroanatomical basis? Trends in Cognitive Sciences, 9, 566-571.

Davidson, R. J. (1993). Cerebral asymmetry and emotion: Conceptual and methodological conundrums. Psychophysiology, 35, 607-614.

Davidson, R. J., \& Irwin, W. (1999). The functional neuroanatomy of emotion and affective style. Trends in Cognitive Sciences, 3, 11-21.

Dietrich, A. (2003). Functional neuroanatomy of altered states of consciousness: The transient hypofrontality hypothesis. Consciousness and Cognition, 12, 231-256.

Dietrich, A. (2006). Transient hypofrontality as a mechanism for the psychological effects of exercise. Psychiatry Research, 145, 79-83.

Ekkekakis, P. (2003). Pleasure and displeasure from the body: Perspectives from exercise. Cognition and Emotion, 17, 213-39.

Ekkekakis, P., \& Acevedo, E. O. (2006). Affective responses to acute exercise: Toward a psychobiological dose-response model. In E. O. Acevedo \& P. Ekkekakis (Eds.), Psychobiology of physical activity (pp. 91-109). Champaign: Human Kinetics.

Ekkekakis, P., Hall, E. E., \& Petruzzello, S. J. (2005). Some like it vigorous: Individual differences in the preference for and tolerance of exercise intensity. Journal of Sport and Exercise Psychology, 27, 350-374.

Ekkekakis, P., Lind, E., Hall, E. E., \& Petruzzello, S. J. (2007). Can selfreported tolerance of exercise intensity play a role in exercise testing? Medicine and Science in Sports and Exercise, 39, 1193-1199.

Ekkekakis, P., Parfitt, G., \& Petruzzello, S. J. (2011). The pleasure and displeasure people feel when they exercise at different intensities: Decennial update and progress towards a tripartite rationale for exercise intensity prescription. Sports Medicine, 41, 641-671.

Ekkekakis, P., \& Petruzzello, S. J. (1999). Acute aerobic exercise and affect: Current status, problems and prospects regarding dose-response. Sports Medicine, 28, 337-374.

Ferrari, M., \& Quaresima, V. (2012). Near infrared brain and muscle oximetry: From the discovery to current applications. Journal of Near Infrared Spectroscopy, 20, 1-14. doi:10.1255/jnirs.973

Gaskill, S. E., Ruby, B. C., Walker, A. J., Sanchez, O. A., Serfass, R. C., \& Leon, A. S. (2001). Validity and reliability of combining three methods to determine the ventilatory threshold. Medicine and Science in Sports and Exercise, 33, 1841-1848.

Goldin, P. R., McRae, K., Ramel, W., \& Gross, J. J. (2008). The neural bases of emotion regulation: Reappraisal and suppression of negative emotion. Biological Psychiatry, 63, 577-586.

Hall, E. E., Petruzzello, S. J., Ekkekakis, P., Miller, P. C., \& Bixby, W. R. (2014). The role of self-reported individual differences in preference for and tolerance of exercise intensity in fitness-testing performance. Journal of Strength and Conditioning Research, 28, 2443-2451.

Hardy, C. J., \& Rejeski, W. J. (1989). Not what, but how one feels: The measurement of affect during exercise. Journal of Sport and Exercise Psychology, 11, 304-317.

Hariri, A. R., Bookheimer, S. Y., \& Mazziotta, J. C. (2000). Modulating emotional responses: Effects of a neocortical network on the limbic system. NeuroReport, 11, 43-48.

Hariri, A. R., Mattay, V. S., Tessitore, A., Fera, F., \& Weinberger, D. R. (2003). Neocortical modulation of the amygdala response to fearful stimuli. Biological Psychiatry, 53, 494-501.

Jung, J., Moser, M., Baucsek, S., Dern, S., \& Schneider, S. (2015). Activation patterns of different brain areas during incremental exercise measured by near-infrared spectroscopy. Experimental Brain Research, 4(233), 4201-4204. 
Ochsner, K. N., \& Gross, J. J. (2008). Cognitive emotion regulation: Insights from social cognitive and affective neuroscience. Current Directions in Psychological Science, 17, 153-158.

Ochsner, K. N., Ray, R. D., Cooper, J. C., Robertson, E. R., Chopra, S., Gabrieli, J. D. E., \& Gross, J. J. (2004). For better or for worse: Neural systems supporting the cognitive down- and up-regulation of negative emotion. NeuroImage, 23, 483-499. doi:10.1016/j. neuroimage.2004.06.030

Perrey, S. (2012). NIRS for measuring cerebral hemodynamic responses during exercise. In H. Boecker, C. H. Hillman, L. Scheef, \& H. K. Strüder (Eds.), Functional neuroimaging in exercise and sport sciences (pp. 335-349). New York: Springer.

Phan, K. L., Fitzgerald, D. A., Nathan, P. J., Moore, G. J., Uhde, T. W., \& Tancer, M. E. (2005). Neural substrates for voluntary suppression of negative affect: A functional magnetic resonance imaging study. Biological Psychiatry, 57, 210-219.

Quirk, G. J., Likhtik, E., Pelletier, J. G., \& Paré, D. (2003). Stimulation of medial prefrontal cortex decreases the responsiveness of central amygdala output neurons. Journal of Neuroscience, 23, 8800-8807.

Robertson, C. V., \& Marino, F. E. (2015). Prefrontal and motor cortex EEG responses and their relationship to ventilatory thresholds during exhaustive incremental exercise. European Journal of Applied Physiology, 115, 1939-1948. doi:10.1007/s00421-015-3177-x

Rooks, C. R., Thom, N. J., McCully, K. K., \& Dishman, R. K. (2010). Effects of incremental exercise on cerebral oxygenation measured by near-infrared spectroscopy: A systematic review. Progress in Neurobiology, 92, 134-150.

Russell, J. A., Weiss, A., \& Mendelsohn, G. A. (1989). Affect Grid: A single item scale of pleasure and arousal. Journal of Personality and Social Psychology, 57, 493-502.

Tempest, G. D., Eston, R., \& Parfitt, G. (2014). Prefrontal cortex haemodynamics and affective responses during exercise: A multichannel near infrared spectroscopy study. PLOS ONE, 9, e95924. doi:10.1371/journal.pone. 0095924

Tempest, G. D., \& Parfitt, G. (2013). Imagery use and affective responses during exercise: An examination of cerebral haemodynamics using near infrared spectroscopy. Journal of Sport and Exercise Psychology, 35, 503-513.

van der Zee, P., Cope, M., Arridge, S. R., Essenpreis, M., Potter, L. A., Edwards, A. D., . . . Delpy, D. T. (1992). Experimentally measured optical pathlengths for the adult head, calf and forearm and the head of the newborn infant as a function of inter optode spacing. In T. K. Goldstick, M. McCabe, \& D. J. Maguire (Eds,), Oxygen transport to tissue $X I I I$ (Advances in Experimental Medicine and Biology, Vol. 316, pp. 143-153). New York, NY: Plenum Press.

Woo, M., Kim, S., Kim, J., Petruzzello, S. J., \& Hatfield, B. D. (2009). Examining the exercise-affect dose-response relationship: Does duration influence frontal EEG asymmetry? International Journal of Psychophysiology, 72, 166-172. 